\title{
Preventing and Controlling COVID-19: A Practical-Based Review in Offshore Workplace
}

\author{
Hendar Sunandar ${ }^{1,2}$, Doni Hikmat Ramdhan ${ }^{1 *}$
}

\begin{abstract}
${ }^{1}$ Department of Occupational Health and Safety, Faculty of Public Health, Universitas Indonesia, Depok, Indonesia
${ }^{2}$ Department of Health, Safety, Security and Environment, PT. Pertamina Hulu Mahakam, Balikpapan, Indonesia
\end{abstract}

\begin{abstract}
An offshore platform is a workplace with complex facilities and limited space due to the complex installed equipment and components. Therefore, the offshore as enclosed area platform is more likely to have a high risk of COVID-19 outbreak. Furthermore, a company must strictly follow health protocols to prevent workers from being exposed to COVID-19 in the offshore workplace. However, workers are often forced to onboard without proper health protocols because of operational needs and production targets. This paper aimed to explore the essence of the steps in preventing and controlling COVID-19 in the offshore workplace and the challenges. The analysis found that the company must take preventive measures against COVID-19 before workers are on board and in the workplace and control it using the hierarchy of control: engineering control, administrative control, and personal protective equipment (PPE).
\end{abstract}

Keywords: COVID-19 prevention, offshore workplace, the hierarchy of control

\section{Introduction}

Since COVID-19 was declared as a global pandemic by the Director-General of the World Health Organization (WHO) on March 11, 2020, the number of confirmed cases of COVID-19 continues to increase. The COVID-19 has impacted industries, including international travel, tourism, hospitality, food and beverages, mining, and small or medium enterprises. ${ }^{1}$ The workers become unsafe because they can be exposed to SARS-CoV-2, a virus that causes COVID-19 and infects co-workers. It was reported that thousands of health workers and other public service workers were exposed to SARS-CoV-2 in the United States of America due to interactions with patients and fellow workers. ${ }^{2}$ Further, 255 million working hours were lost in 2020 due to COVID-19. ${ }^{3}$ Therefore, the COVID-19 pandemic has impacted workers and productivity.

Limited space (enclosure area), such as in oil and gas offshore platforms and supporting boats, are vulnerable sites of COVID-19 outbreak due to their high person on board (POB) density, constrained internal workplace and accommodation, and relatively concentrated mess hall. ${ }^{4}$ Offshore facilities are complex workplaces due to their large diversity of equipment and components that should be installed for drilling activities, oil and gas processes, and accommodation. ${ }^{5}$ The accommodation in offshore

Correspondence $^{*}$ : Doni Hikmat Ramdhan, Department of Occupational Health and Safety, Faculty of Public Health, Universitas Indonesia, C Building 1st Floor Kampus Baru UI Depok 16424, Indonesia, E-mail: doni@ui.ac.id, Phone: +62 85697968639 facilities is highly airtight. Cabins are divided into workers' accommodations and public areas, such as mess hall, galley, recreation room, gym room, meeting room, and praying room. A worker might be exposed to COVID-19 in an offshore workplace due to close contact (less than 1.8 meters) with other workers and touching their mouth, nose, and eyes after frequently contacting touched surfaces and work equipment. ${ }^{6}$ Consequently, the offshore workplace is among the workplaces prone to COVID-19 outbreak.

The company is obligated by law to provide and maintain a safe workplace from any hazards, such as a virus that causes COVID-19.7 Measures must be taken to minimize the probability of viruses' exposure to the workers and avoid the virus spread from an already exposed worker. ${ }^{8}$ Thus, protection against COVID-19 transmission in the enclosure workplace, such as offshore platforms, must be carried out through comprehensive and systematic prevention and controlling efforts before onboard and at the workplace. The main goal is to minimize the health risk in the workplace, if not eliminated.

This paper presented an article review to explore the essence of preventing the COVID-19 outbreak in the offshore workplace and controlling it using a hierarchy of control.

Received : June 9, 2021

Accepted : July 23, 2021

Published: July 31, 2021 


\section{Method}

Related articles in preventing and controlling COVID-19 at the workplace, especially practical-based studies on the offshore platform, were searched and analyzed. The discussion was then divided into three categories: prevention, control, and challenges. All best practices from the WHO and Centers for Disease Control and Prevention (CDC) were also considered. Furthermore, the steps in preventing and controlling COVID-19 in the offshore workplace were identified.

\section{Results and Discussion}

\section{COVID-19 Prevention in Offshore Workplace}

The first and most crucial aspect of preventing COVID-19 at the offshore workplace is pre-employment and periodic medical check-ups (MCUs). A company doctor reviews the results to determine whether the workers are fit for work, following the minimum requirements for each type of job position (job risk base).${ }^{9} \mathrm{MCU}$ is a clinical preventive service carried out on workers lacking signs and symptoms of disease 8 to ensure that only healthy workers can work according to their roles and responsibilities. MCU implementation is part of the company and employee compliance with Law No. 1 of 1970 concerning Occupational Safety, Government Regulation of the Republic of Indonesia No. 50 of 2012 concerning Implementation of Occupational Health and Safety Management Systems (OHSMS), and Regulation of the Minister of Manpower and Transmigration Number Per.02/Men/1998 concerning Health Examination for Workers in the Implementation of Occupational Health and Safety. The types of examination parameters are determined according to the risk of each job. ${ }^{9-11}$ The final results of the MCU become the baseline data for further health assessments related to COVID-19 comorbidities.

Several diseases, such as diabetes mellitus, chronic obstructive pulmonary disease (COPD), cardiovascular disease, hypertension, liver disease, obesity, kidney disease, and malignancy, are COVID-19 comorbidities. ${ }^{12}$ Workers with comorbidities have a higher susceptibility to COVID-19, more severe complications, and even death. ${ }^{13}$ Therefore, the company must determine that every comorbid worker can only work from home (WFH). The company can grant work permit exemptions to essential workers whose physical presence is necessary to maintain company business continuity. For this purpose, the company must first conduct a risk assessment and mitigation plan (RAMP) and medical recommendations for workers with comorbidities. Supervisors must ensure that workers with comorbidities permitted to work will follow the RAMP document's recommendations. Companies can give warnings to workers who do not follow health recommendations or revoke their work permits.

The company needs to facilitate workers in carrying out a self-health evaluation before onboard to offshore. 14 Self-health evaluation can be done through an online questionnaire or application to assess potential COVID19 exposure before onboard. The company with several workers who live outside the company's operational area, such as workers in mining, oil, and gas companies with on-and-off schedules, where some of them travel by themselves using public transportation modes (air or land) from their domicile to the company premises, must provide a quarantine facility for at least one day before antigen-detecting rapid diagnostic tests (Ag-RDTs) test ${ }^{15}$ or seven days before reverse transcription-polymerase chain reaction (RT-PCR) test. ${ }^{16}$

A COVID-19 test must be conducted on every worker with no potential exposure to SARS-CoV-2 based on the health self-evaluation. The COVID-19 test is conducted as part of programs to reduce COVID-19 spread, especially for travelers and those entering work areas, such as the offshore workplace. ${ }^{17}$ COVID-19 test is effective in preventing COVID-19 in the workplace. However, some people with COVID-19 have no symptoms (asymptomatic) or are still in the incubation period, making it difficult to identify them by physical examination. ${ }^{18}$ Body temperature screening and self-health assessment must be performed daily when they are at the offshore workplace.

The COVID-19 test by nucleic acid amplification test (NAAT), such as RT-PCR, is highly recommended because of its high sensitivity ${ }^{19,20}$ for workers working in enclosure areas, such as oil and gas offshore platforms. A negative result is required to get a permit to work offshore. ${ }^{20} \mathrm{Ag}$-RDTs with a sensitivity of $94 \%$, slightly lower than RT-PCR, are the second choice with lower cost and faster results. Ag-RDTs can also diagnose COVID19 when RT-PCR assays are unavailable. ${ }^{19}$ Antibody assay (serology) with low sensitivity is the last option when RT-PCR and AgRDTs are unavailable. ${ }^{19}$ A positive IgM titer from an antibody test reflects an acute (current) infection, while a positive IgG titer indicates a previous infection. ${ }^{19,20}$

Workers' transportation to and from the offshore workplace is the next critical point. Public transportation from home to jetty is permitted only when health protocols can be implemented, such as maintaining physical distance, providing barriers/partitions between drivers and passengers, and disinfecting after each use. The use of one's own transportation is highly recommended. If resources are sufficient, the company can provide transportation for all workers from home to jetty. If the company offers transportation, then the seats must be arranged at a minimum distance of one meter. The frequency of transportation must be adjusted because the 
number of workers in one vehicle is reduced. 21 Partitions/barriers between the driver and passengers must be provided; workers must wear masks and forbid talking to each other and disinfect their vehicle after each use. Disinfection is necessary because one of the SARS$\mathrm{CoV}-2$ characteristics is that it can survive on inanimate surface objects for 2-9 days at room temperature. ${ }^{22}$ Passengers' name logs and seat numbers must be recorded to anticipate if a confirmed case of COVID-19 is found, so it will be easy to trace close contacts from one vehicle.

A protocol for managing confirmed COVID-19 cases needs to be drawn up to ensure proper handling if confirmed cases of COVID-19 are found in the workplace and to trace their close contacts. Every worker who has symptoms of COVID-19 must be immediately isolated and confirmed by the NAAT test at the nearest health facilities. Standard operational procedures to evacuate infected workers to the closest health facilities should be established to ensure that there is no exposure to other workers. Companies must provide temporary isolation and quarantine facilities at the workplace, especially if the workplace is far from healthcare facilities such as offshore oil and gas and coal mining. Temporary isolation and quarantine rooms must be equipped with N95 masks, telephones, water for bathing and washing, latrines, disposable soaps and tissues, and medical telephone numbers for emergency contact. ${ }^{14}$

Close contact tracing should be carried out immediately by involving the relevant functions/departments through an interview with confirmed caseworkers and their co-workers or CCTV footage if available in the workplace. The transmission of COVID-19 starts about two days before workers show symptoms, so close contact tracing must be carried out from two days before symptoms until 14 days after the onset of symptoms. For confirmed cases of COVID-19 with no symptoms, close contact tracing is carried out from three days before NAAT sampling until 14 days after. This procedure considers the average incubation period of COVID-19 at 5.2 days and a maximum of 14 days. ${ }^{23}$

Through related functions, such as the occupational health function, the company must monitor every confirmed case of COVID-19, probable case, suspected case, and close contact. Heads of related functions can coordinate with workers to ensure compliance with quarantine or isolation protocols. Confirmed case monitoring is imperative as part of the return to work assessment. Workers who have completed the quarantine and isolation period with a negative NAAT test can then be allowed to return to work.

\section{COVID-19 Control in Offshore Workplace}

Control of COVID-19 in the offshore workplace can be developed using a control hierarchy. The elimination of SARS-CoV-2 is more useful than other control measures. However, it may be complicated, even impossible, to eliminate or substitute COVID-19 from the workplace. ${ }^{24}$ The engineering controls, administrative controls, PPE, or the combination are the most effective ways to control COVID-19 in the offshore workplace. Engineering controls are aimed at isolating the worker from the hazard of SARS-CoV-2. Workstations in the offshore workplace need to be adjusted so that workers do not face each other. If possible, physical barriers can be established to ensure that workers are not in close contact with others, especially in public areas such as workshops, coffee break areas, galley, restroom, locker room, meeting room, and entrance/exit. Physical barriers/partitions can be made of strip curtains, plastic barriers, or similar materials as an impermeable divider.6,14 Considering that offshore has limited space, it is necessary to signal worker traffic in a single direction in narrow areas, such as stairwells, aisles, incoming and outgoing registration points, entrance/exit doors, and entrance/exit helideck. Physical distance signs must be created in most public areas, such as shared workstations, galley, and coffee break areas. 6

Handwashing stations with soap or hand sanitizer dispensers that contain at least $60 \%$ alcohol should be provided in many work and public areas at offshore facilities. WHO and US CDC recommend frequent handwashing as their topmost COVID-19 prevention advice for the public. ${ }^{25}$ Frequent handwashing with soap and alcoholbased hand rub is proven to be the best and most effective way to reduce the spread of respiratory infection. 26,27

Another critical point as part of engineering control is to ensure that offshore facilities have good ventilation by maintaining the maximum amount of fresh air delivered to the workplace and keeping humidity at a level of $40 \%-60 \%$. Most of the spread of respiratory diseases can be reduced in any circumstance by good air ventilation. ${ }^{28,29}$ Heating, ventilation, and air conditioning (HVAC) filters can be increased to the highest acceptable level. Also, portable high-efficiency particulate air (HE-PA) filtration units can be installed to remove any biological contaminants in the air when open ventilation is not well established.

For administrative control, it is important to minimize the exposure of SARS-CoV-2 to workers in the offshore workplace by changing work procedures. Online meetings and shift arrangements are part of administrative control to avoid face-to-face interaction and communication. Training and written company policies in controlling COVID-19 are other administrative controls that the company must carry out. The training may be focused on physical distancing, frequent handwashing, wearing appropriate PPE, and how to dispose of used masks. ${ }^{24}$ 
The last control uses PPE as an integrated part of COVID-19 control strategies in the offshore workplace. Wearing a 3-ply cloth mask is considered sufficient for most workers, while others will need additional PPE based on their risks, such as respirators, face shields, goggles, gloves, and gowns. A health worker offshore (medical doctor or nurse) must wear all the PPEs mentioned above because of their risk when handling patients. All types of PPE must be inspected, maintained, and used properly to provide adequate protection to users.

\section{Challenges in Preventing and Controlling COVID-19}

Limited facilities for self-quarantine for workers before screening tests and before onboarding and the facilities for self-isolation for confirmed caseworkers are often a challenge in COVID-19 prevention and control programs. In addition to costs, this problem arises because of the limited area to add isolation and quarantine rooms at company premises. Alternatively, the company can work with hotel service providers to provide self-isolation and self-quarantine rooms upon approval from local health authorities. However, when self-quarantine rooms before on board are equipped at company facilities, it is common to be an issue because quarantine will increase the number of days workers spend.

The next challenge is workers' compliance with implementing the health protocol during self-quarantine before onboard. Workers are required to carry out 14 days of self-quarantine at home before onboard. Note that the company cannot fully control the quarantine protocols carried out by workers and has not provided room for self-quarantine in company facilities that can be monitored. Providing a surveillance application with geotagging is one solution, but it needs to be appropriately communicated to every worker because of the possibility of resistance related to privacy issues.

\section{Conclusion}

Preventing and controlling COVID-19 in the offshore workplace is essential for a company's business continuity. Periodic MCUs can carry out the COVID-19 prevention, comorbid review, self-health evaluation, self-quarantine, and COVID-19 test before onboard, arrangement of workers' transportation modes, case management of confirmed caseworker, probable case, suspected case, and close contacts in the workplace. The COVID-19 control can be carried out through a hierarchy of control. The elimination is more useful than other controls, but it may be difficult or even impossible. The most effective ways are engineering control, administrative control, PPE, and their combination.

\footnotetext{
Abbreviations

Ag-RDTs: Antigen-detecting rapid diagnostic tests; COVID-19:

Coronavirus Disease 2019; MCU: Medical Check-Up; NAAT: Nucleic Acid Amplification Test; SARS-CoV: Severe Acute Respiratory Syndrome Coronavirus; OHSMS: Occupational Health and Safety Management Systems; PPE: Personal Protection Equipment; RAMP: Risk Assessment and Mitigation Plan; RT-PCR: Reverse Transcription Polymerase Chain Reaction; WFH: Work from Home.

\section{Ethics Approval and Consent to Participate}

No ethics approval since this article is not experimental research.

\section{Competing Interest}

The authors declare that no competing financial interest, professional, or personal interest affected the manuscript.

\section{Availability of Data and Materials}

No additional data and materials are available.

\section{Authors' Contribution}

HS and DHR were involved in the conception of the manuscript. HS wrote the first draft, then reviewed and edited by DHR. Both HS and DHR read and approve the final manuscript.

\section{Acknowledgment}

This research was supported by Universitas Indonesia, Directorate of Research and Community Engagement (PUTI grant number 48592020).

\section{References}

1. Aguiar-Quintana T, Nguyen H, Araujo-Cabrera Y, Sanabria-Díaz JM. Do job insecurity, anxiety and depression caused by the COVID-19 pandemic influence hotel employees' self-rated task performance? The moderating role of employee resilience. International Journal of Hospitality Management. 2021; 94: 1-9.

2. Michaels D, Wagner GR. Occupational Safety and Health Administration (OSHA) and worker safety during the COVID-19 pandemic. Jama. 2020; 324(14): 1389-90.

3. International Labour Organization. COVID-19 and the world of work, 3rd ed. 2020; 1-23.

4. Liu X, Chang Y-C. An emergency responding mechanism for cruise epidemic prevention - taking COVID-19 as an example. Marine Policy. 2020; 119: 104093.

5. Chandrasekaran S. Dynamic analysis and design of offshore structures. New Delhi: Springer. 2015 p. 3.

6. Centers for Disease Control and Prevention. COVID-19 employer information for offshore oil and gas; 2020.

7. George R, George A. Prevention of COVID-19 in the workplace. South African Medical Journal. 2020; 110(4): 1.

8. Cirrincione L, et al. COVID-19 pandemic: prevention and protection measures to be adopted at the workplace. Sustainability. 2020; 12(9): 3603.

9. Maersk G, Goodge A, McKechnie R. Medical aspects of fitness for offshore work: guidance for examining physicians. London: Oil \& Gas UK. 2008 p. 1-2.

10. Culica D, Rohrer J, Ward M, Hilsenrath P, Pomrehn P. Medical checkups: who does not get them?. American Journal of Public Health. 2002; 92(1): 88-91. 
11. Palmer KT, Brown I, Hobson J. Fitness for work: the medical aspects, 5th ed. Oxford: Oxford University Press. 2019 p. 1-20.

12. Ejaz H, Alsrhani A, Zafar A, Javed H, Junaid K, Abdalla AE, et al. COVID-19 and comorbidities : deleterious impact on infected patients. Journal of Infection and Public Health. 2020; 13(12): 1833-39.

13. Jakhmola S, Indari O, Baral B, Kashyap D, Varshney N, Das A, et al. Comorbidity assessment is essential during COVID-19 treatment. Frontiers in Pshysiology. 2020; 11: 984.

14. Offshore Operators Committee. COVID-19 management strategies for offshore energy operations; 2020.

15. Centers for Disease Control and Prevention. Testing overview; 2021.

16. Cevik M, Tate M, Lloyd O, Maraolo AE, Schafers J, Ho A. SARS-CoV2, SARS-CoV, and MERS-CoV viral load dynamics, duration of viral shedding, and infectiousness: a systematic review and meta-analysis. The Lancet Microbe. 2020; 5247(20): 1-10.

17. Gostic K, Gomez ACR, Mummah RO, Kucharski AJ, Lloyd-Smith JO. Estimated effectiveness of symptom and risk screening to prevent the spread of COVID-19. Epidemiology and Global Health. 2020; 9: e55570.

18. Quilty BJ, Clifford S, Flasche S, Eggo RM. Effectiveness of airport screening at detecting travellers infected with novel coronavirus (2019-nCoV). Eurosurveillance. 2020; 25(5): 2000080.

19. World Health Organization. Public health surveillance for COVID-19: interim guidance; 2020.

20. Rueda-Garrido JC, Vicente-Herrero MT, Del Campo MT, ReinosoBarbero L, de la Hoz RE, Delclos GL, et al. Return to work guidelines for the COVID-19 pandemic. Occupational Medicine. 2020; 70(5): 300-05.
21. Gkiotsalitis K, Cats O. Optimal frequency setting of metro services in the age of COVID-19 distancing measures. Transpormetrica: Transport Science. 2021; 1-21.

22. Kampf G, Todt D, Pfaender S, Steinmann E. Persistence of coronaviruses on inanimate surfaces and their inactivation with biocidal agents. Journal of Hospital Infection. 2020; 104(3): 246-51.

23. Wu YC, Chen CS, Chan YJ. The outbreak of COVID-19: an overview. Journal of the Chinese Medical Association. 2020; 83(3): 217-20.

24. Dehghani F, Omidi F, Yousefinejad S, Taheri E. The hierarchy of preventive measures to protect workers against the COVID-19 pandemic: a review. Work. 2020; 67(4): 771-7.

25. Ray I. Viewpoint - Handwashing and COVID-19: simple, right there...? World Development. 2020; 135: 105086.

26. Gould DJ, Moralejo D, Drey N, Chudleigh JH, Taljaard M. Interventions to improve hand hygiene compliance in patient care. Cochrane Database of Systematic Reviews. 2017; (9).

27. Jefferson T, Del Mar CB, Dooley L, Ferroni E, Al-Ansary LA, Bawazeer GA, et al. Physical interventions to interrupt or reduce the spread of respiratory viruses. Cochrane Database of Systematic Reviews. 2020; (11): 1-310.

28. Sun C, Zhai Z. The efficacy of social distance and ventilation effectiveness in preventing COVID-19 transmission. Sustainable Cities and Society. 2020; 62: 102390.

29. Yang J, Sekhar SC, Cheong KWD, Raphael B. Performance evaluation of a novel personalized ventilation-personalized exhaust system for airborne infection control. Indoor Air. 2015; 25(2): 176-87. 